\title{
A Procedure to Calibrate Multiparameter Weather Radar Using Properties of the Rain Medium
}

\author{
Eugenio Gorgucci, Gianfranco Scarchilli, and V. Chandrasekar, Member, IEEE
}

\begin{abstract}
The joint distribution characteristics of size and shape of raindrops directly translate into features of polarization diversity measurements in rainfall. Theoretical calculations as well as radar observations indicate that the three polarization diversity measurements, namely, reflectivity, differential reflectivity, and specific differential propagation phase, lie in a constrained space that can be approximated by a three-dimensional (3-D) surface. This feature as well as the vertical-looking observation of raindrops are used to determine biases in calibration of the radar system. A simple procedure is developed to obtain the bias in the absolute calibration from polarization diversity observation in rainfall. Simulation study as well as data analysis indicate that calibration errors can be estimated to an accuracy of $1 \mathrm{~dB}$.
\end{abstract}

Index Terms - Calibration, meteorological radar, polarimetric radar.

\section{INTRODUCTION}

$\mathbf{T}$ HE characteristics of polarization diversity measurements in rain are determined by the shape and size distribution of raindrops. The shape of a raindrop is determined by the forces due to the surface tension and hydrostatic and aerodynamic pressures due to airflow around the raindrop. Medium and large raindrops ( $>2 \mathrm{~mm}$ in diameter) are nonspherical. The shape of raindrops changes with the size and can be approximately described by an oblate spheroid with the symmetry axis in the vertical direction [1], [2], [6]. The properties of the size-shape relationship of raindrops translate directly into features of polarization diversity measurements in rainfall. Theoretical calculation and radar observations suggest that polarization diversity measurements of rain, namely, 1) radar reflectivity factor $\left.\left(Z_{H}\right), 2\right)$ differential reflectivity $\left(Z_{D R}\right)$, and 3) specific differential phase $\left(K_{D P}\right)$ lie in a limited threedimensional (3-D) space [3]. This constraint can be utilized to study the calibration errors in a multiparameter weather radar [4].

In principle, it can be stated that the calibration errors in a multiparameter radar can be corrected by enforcing the constraints of observations in rain. However, in practice, the technique to calibrate the multiparameter radar using this self-

Manuscript received December 9, 1996; revised December 16, 1997. This work was supported in part by the National Group for Defence from Hydrogeological Hazards (CNR, Italy), by Mesoscale Alpine Project (MAPCNR), and by NSF (ATM-9 413453).

E. Gorgucci and G. Scarchilli are with the Istituto di Fisica dell'Atmosfera (CNR), Area di Ricerca Roma-Tor Vergata, 00133 Rome, Italy (e-mail: gorgucci@radar.ifa.rm.cnr.it).

V. Chandrasekar is with Colorado State University, Fort Collins, CO 80523 USA.

Publisher Item Identifier S 0196-2892(99)00041-8. consistency procedure is not straightforward and numerous details are important This paper presents the practical procedure to calibrate multiparameter weather radars. The paper is organized as follows. Section II describes the multiparameter radar measurements in rainfall and their self-consistency. In Section III, the issues involved in calibrating differential reflectivity are described. Section IV presents the procedure to correct errors in absolute calibration. Section V summarizes the results of this paper.

\section{SElf-CONSISTENCY OF POLARIZATION DIVERSITY OBSERVATION IN RAIN}

Cloud models and measurements of raindrop size distribution (RSD) at the surface and aloft show that a gamma distribution model adequately describes many of natural variations in the RSD [5]

$$
N(D)=n_{C} f_{D}(D) \quad\left(\mathrm{m}^{-3} \mathrm{~mm}^{-1}\right)
$$

where $N(D)$ is the number of raindrops per unit volume per unit size interval ( $D$ to $D+\Delta D), n_{C}$ is the concentration, and $f_{D}(D)$ is the gamma probability density function (pdf). $f_{D}(D)$ can be written as

$$
f_{D}(D)=\frac{\Lambda^{\mu+1}}{\Gamma(\mu+1)} e^{-\Lambda D} D^{\mu}
$$

where $\mu$ and $\Lambda$ are parameters of the distribution. The median drop diameter $D_{0}$ can be obtained in terms of the parameters of the distribution as

$$
D_{0}=\frac{3.67+\mu}{\Lambda}
$$

The shape of a raindrop can be approximated by an oblate spheroid with the axis ratio $(b / a)$ given by the empirical relation [6]

$$
\frac{b}{a}=1.03-0.062 D
$$

where $D$ is the equivolumetric spherical diameter of a raindrop in millimeters and $a$ and $b$ are the semimajor and semiminor axis of the raindrop. The radar reflectivity factor of the rain medium can be expressed in terms of the RSD as

$$
Z_{H, V}=\frac{\lambda^{4}}{\pi^{\tilde{5}}|K|^{2}} \int \sigma_{H, V}(D) N(D) d D \quad\left(\mathrm{~mm}^{6} \mathrm{~m}^{-3}\right)
$$

where $Z_{H, V}$ and $\sigma_{H, V}$ represent the reflectivity factors and radar cross sections at horizontal $(\mathrm{H})$ and vertical $(\mathrm{V})$ polarizations, respectively, $\lambda$ represents the wavelength, and 
$K=\left(\epsilon_{r}-1\right) /\left(\epsilon_{r}+2\right)$, where $\epsilon_{r}$ is the dielectric constant of water. Similarly differential reflectivity $\left(Z_{D} R\right)$ and specific differential phase $\left(K_{D} P\right)$ can be obtained as

$$
\begin{aligned}
Z_{D R}= & \frac{\int \sigma_{H}(D) N(D) d D}{\int \sigma_{V}(D) N(D) d D} \\
K_{D P}= & \frac{180 \lambda}{\pi} \operatorname{Re} \int\left[f_{H}(D)-f_{V}(D)\right] N(d) \\
& \left.\cdot d D(\operatorname{deg~km})^{-1}\right)
\end{aligned}
$$

where $f_{H}$ and $f_{V}$ are the forward scatter amplitudes at $\mathrm{H}$ and V-polarization, respectively. Scarchilli et al. [3] have shown that multiparameter radar observations in rain, namely, $Z_{H}, Z_{D R}$ and $K_{D P}$, vary over a constrained 3-D space. This constraint enables parameterization of $K_{D P}$ in terms of $Z_{H}$ and $Z_{D R}$ as

$$
K_{D P}^{*}=C Z_{H}^{\alpha} 10^{-\beta Z_{D R}}
$$

where $K_{D P}^{*}$ is the parameterized estimate of $K_{D P}, Z_{H}$ is in units $\mathrm{mm}^{6} \mathrm{~m}^{-3}$, and $Z_{D R}$ is in decibels. It should be noted here that $K_{D P}^{*}$ indicates an estimate based on $Z_{H}$ and $Z_{D R}$ and not from a profile of differential phase measurements. The coefficients $C, \alpha$, and $\beta$ change as a function of the frequency. The values of $C, \alpha$, and $\beta$ at $S$ - and $C$-bands are given as follows.

$S$-band $(10 \mathrm{~cm})$

$$
C=1.05 \times 10^{-4} \quad \alpha=0.96 \quad \beta=0.26 .
$$

$C$-band $(5.5 \mathrm{~cm})$

$$
C=1.46 \times 10^{-4} \quad \alpha=0.98 \quad \beta=0.20 .
$$

Scarchilli et al. [3] have shown that (8) can approximate $K_{D P}$ fairly well in the absence of measurement errors. Thus, we can see that $Z_{H}, Z_{D R}$, and $K_{D P}$ exist in a constrained 3-D space and (8) represents a 3-D surface approximation of the variability of $Z_{H}, Z_{D R}$, and $K_{D P}$. In the following section, this self-consistency feature is utilized to study biases in calibration of multiparameter radars.

\section{VERTICAL-LOOKING OBSERVATION OF $Z_{D R}$ AND ITS CALIBRATION}

The shape of raindrops can be approximated by oblate spheroids. Radar and in situ aircraft-based observations show that on average the raindrops are oriented with the symmetry axis in the vertical direction. This implies that the shape of raindrops seen at an elevation angle of $90^{\circ}$ is nearly circular. Therefore, $Z_{D R}$ measurements performed with the antenna pointing at an elevation of $90^{\circ}$ should be $0 \mathrm{~dB}$. There can be deviations from the $0-\mathrm{dB}$ value of $Z_{D R}$ due to the following reasons:

1) mean canting angle of raindrops different from zero;

2) ground clutter contribution;

3) system bias given by unequal paths or gains in the Hand V-polarized channels.
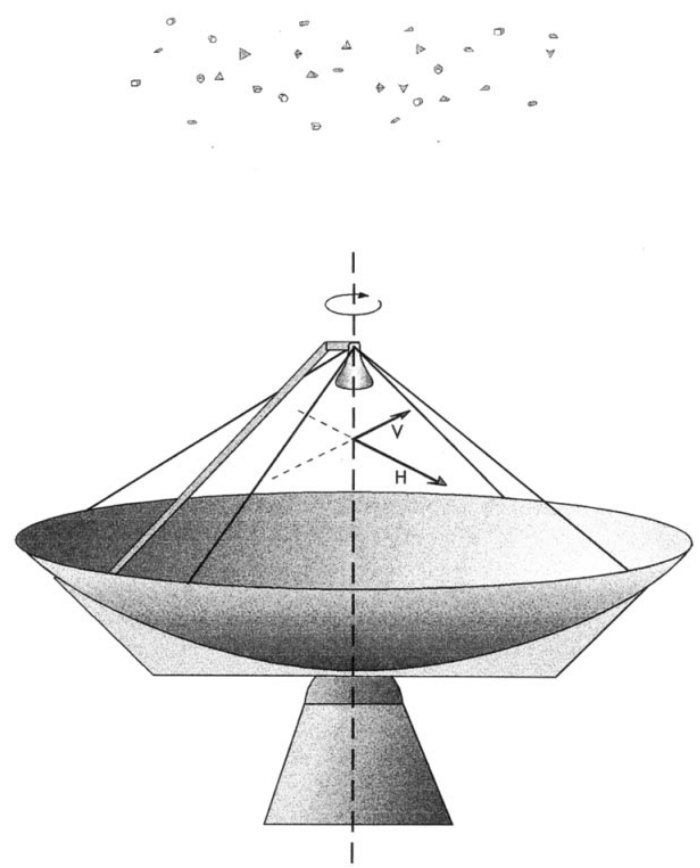

Fig. 1. Variation of $\mathrm{H}$ - and V-polarization directions due to the rotation of the radar antenna about the vertical.

Items 1) and 2) depend on $\mathrm{H}$ - and V-polarization directions (see Fig. 1). The average $Z_{D R}$ taken over all possible orientations of $\mathrm{H}$ - and $\mathrm{V}$-polarizations, with the antenna looking vertical for items 1) and 2), go to zero. However if there is nonzero $Z_{D R}$ due to the system bias, it does not change with the different $\mathrm{H}$ and $\mathrm{V}$ orientations looking vertical. In many radars, the different $\mathrm{H}$ and $\mathrm{V}$ orientations can be achieved by changing the azimuth positioning over zero to $360^{\circ}$, keeping the elevation angle at $90^{\circ}$. However, the exact procedure depends on the set up of the scanning servo system of the radar. In summary, the average $Z_{D R}$ computed with all possible orientations of the polarization states of the radar antenna pointing in the vertical direction should be zero. Any nonzero observation can be directly attributed to the radar system bias between the two polarization channels. In the following, we show the estimation of the system $Z_{D R}$ bias using verticallooking data from the NCAR, CP-2 radar, taken over the Kennedy Space Center (KSC), FL, during the Convection and Precipitation/Electrification Experiment (CaPE) [7]. CaPE was conducted in the Central Florida region during the summer of 1991.

Fig. 2 shows the azimuthal profile of $Z_{D R}$ obtained during the rotation of radar antenna about the vertical; each value of $Z_{D R}$ represents an average of $2 \mathrm{~km}$ in altitude and $5^{\circ}$ in azimuth. When the azimuth angle in horizontal axis exceeds $360^{\circ}$, the exact position of the antenna can be obtained subtracting multiples of $360^{\circ}$ from the indicated angle in Fig. 2. We can see from Fig. 2 that the measured $Z_{D R}$ has a periodic structure in the azimuth, with a periodicity of $180^{\circ}$. The mean level of the $Z_{D R}$ value is about $0.15 \mathrm{~dB}$. The full excursion of the $Z_{D R}$ profile as a function of the azimuth is 0.3 $\mathrm{dB}$. This excursion is due to the variability in the ground clutter response with the rotation of the antenna. Vertical-looking 


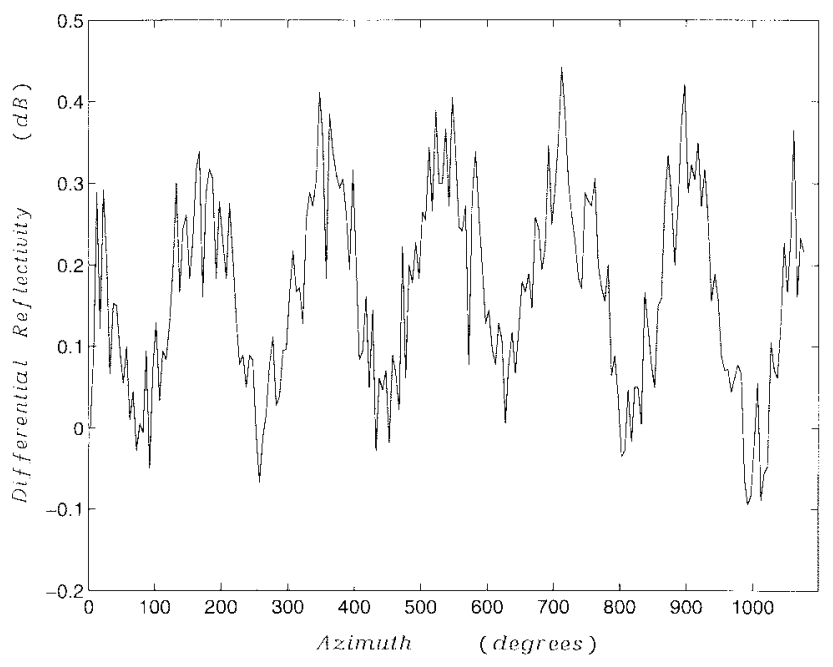

Fig. 2. Profile of vertical-looking $Z_{D R}$ measurements, collected in rain medium below $3.5 \mathrm{~km}$ in altitude, as a function of the azimuth for the radar antenna in continuous rotation about the vertical.

radar observations were made for several minutes, rotating continuously about the vertical. Based on the observations from Fig. 2, we can see that it is important to average $Z_{D R}$ over full cycles of $360^{\circ}$ in azimuth to avoid bias due to the direction-dependent response. The $Z_{D R}$ profile was obtained from data below $3.5 \mathrm{~km}$ in altitude to stay in rain medium. The same profile was observed at various altitudes, and the estimated mean does not change as a function of height, again confirming that the mean $Z_{D R}$ is the system bias. The standard deviation of $Z_{D R}$ evaluated from the data was $0.3 \mathrm{~dB}$. The total number of points used in the mean estimates was about 10000 . Therefore, the standard deviation in the estimate of the $Z_{D R}$ bias is $0.003 \mathrm{~dB}$.

\section{Absolute Calibration}

The absolute calibration of the radar is important for quantitative application of radar measurements, such as rainfall rate estimation. The absolute calibration of the radar depends on several factors, such as the antenna gain, gain of amplifiers, loss in waveguides, etc. In this section, we describe a procedure for using the properties of the rain medium to obtain absolute calibration of the radar. The backscattered mean power received by the radar $\left(P_{r}\right)$ from a resolution volume can be written as

$$
P_{r}=C \frac{Z}{R^{2}}
$$

where $Z$ is the reflectivity factor, $R$ is the range to the volume, and $C$ is the radar constant. Accurate knowledge of $C$ is very important for estimating $Z$ from the received power. Typically the radar constant is known within couple of decibels from past calibration. This calibration may drift by a few decibels due to changes in the properties of the various amplifiers and mixers along the line. Invoking the principle of self-consistency, we can estimate the drift in the calibration of the radar constant. In the absence of measurement errors, a scatter diagram of measured $K_{D P}$ and $K_{D P}^{*}$ obtained from (8) should lie about a 1:1 line. However, in the presence of a bias in the absolute gain of the radar system, a scattergram of $K_{D P}$ versus $K_{D P}^{*}$ will deviate from a 1:1 line. The slope $s$ of this scattergram depends on the amount of bias in the absolute calibration, and it can be obtained as a simple expression based on (8) as

$$
\text { Bias }=10.4 \times \log (s) \quad(\mathrm{dB}) .
$$

The standard deviation of the bias can be approximated by

$$
\sigma(\mathrm{Bias})=4.52 \frac{\sigma(s)}{E(s)} \quad(\mathrm{dB})
$$

where $\sigma(s)$ and $E(s)$ are the standard deviation and the mean value of the slope, respectively.

In the following sections, we present theoretical studies as well as simulations that evaluate the accuracy of the calibration procedure.

\section{A. Estimation of the Bias in Calibration}

The multiparameter measurements in rainfall, namely, $Z_{H}, Z_{D R}$, and $K_{D P}$, lie in a constrained 3-D space that can be approximated by a surface given by (8). $K_{D P}$ is the slope of the differential phase profile with range and is independent of the calibration of the radar. $Z_{D R}$ is a relative power measurement and can be calibrated fairly accurately, as shown in the previous section. Any bias in the absolute calibration of the radar (error in the assumed radar constant) will result in a biased value of reflectivity. This bias will also produce a discrepancy in the self-consistency of the triplet of multiparameter radar measurements $Z_{H}, Z_{D R}$, and $K_{D P}$.

The principle for obtaining the bias in calibration is fairly straightforward. However, numerous details are important to obtain the bias. Firstly, the measurement error and nature of $Z_{H}, Z_{D R}$, and $K_{D P}$ are very different. $Z_{H}$ and $Z_{D R}$ are power measurements, whereas $K_{D P}$ is derived from $\Phi_{D P}$, which is the differential phase measurement. $Z_{H}$ and $Z_{D R}$ are point measurements, whereas $K_{D P}$ is evaluated over a propagation path. A mean $K_{D P}$ value is evaluated typically over a path of few kilometers in length. The measurement error in $K_{D P}$ depends on the number of the range gates along the path, the range resolution, and the profile of $\Phi_{D P}$. In this section, we analyze various profiles of $Z_{H}, Z_{D R}$, and $\Phi_{D P}$ to study the implication on the estimation of bias in calibration. Scarchilli et al. [3] have shown that parameterized $\Phi_{D P}^{*}$ profile can be constructed from measurement of $Z_{H}$ and $Z_{D R}$ using (8). Any error in absolute calibration will result in a deviation from the measured profile of $\Phi_{D P}$, where the difference between $\Phi_{D P}$ and $\Phi_{D P}^{*}$ will progressively increase with range. The actual $\Phi_{D P}$ profile depends on the $\Phi_{D P}$ value at the starting range bin $\left(\Phi_{D P}^{\circ}\right)$, which is the differential phase due to radar system. Therefore, if $\Phi_{D P}^{o}$ is not known accurately, there will be a constant shift between $\Phi_{D P}$ and $\Phi_{D P}^{*}$ profiles, even in the absence of any calibration bias. $\Phi_{D P}^{o}$ can vary over several degrees within a day, as shown in the histogram of Fig. 3, where it can be seen that $\Phi_{D P}^{\circ}$ varies by $10^{\circ}$. We note here that this spread of $10^{\circ}$ includes spread due to measurement errors [8]. Any technique that matches profiles of $\Phi_{D P}$ and $\Phi_{D P}^{*}$ will be affected by uncertainty in the knowledge of initial $\Phi_{D P}^{O}$. Therefore, to overcome this 


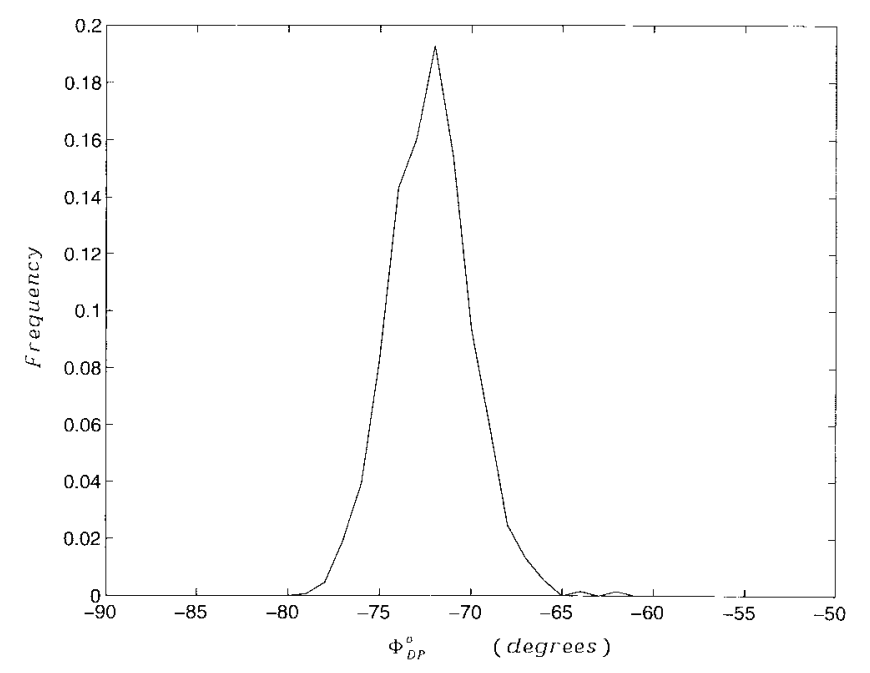

Fig. 3. Histogram of the differential profile phase shift $\Phi_{D P}^{o}$ at the starting range bin.

problem, the range variation of $\Phi_{D P}$ and $\Phi_{D P}^{*}$ profiles is utilized in our analysis.

Finally, we want to point out that important physical factors, such as attenuation and hail contamination, can affect the accuracy of the method. For the calibration purpose, we should avoid using hail-contaminated data because the procedure is strictly for rain medium. As for the issue of attenuation, we can note that, according to (8), the bias $B_{H}$ in $Z_{H}$ is equivalent to the bias $B_{D R}$ in $Z_{D R}$ as

$$
B_{D R}=0.1 \frac{\alpha}{\beta} B_{H} \quad(\mathrm{~dB}) .
$$

Thus, to ensure the accuracy of $Z_{H}$ of $1 \mathrm{~dB}$, we need to have $B_{D R}$ below $0.4 \mathrm{~dB}$ at $S$-band and $0.5 \mathrm{~dB}$ at $C$-band. The bias $B_{D R}$ due to differential attenuation is proportional to the total differential phase and can be approximated as

$$
B_{D R}=b \Phi_{D P} \quad(\mathrm{~dB})
$$

where $b$ is 0.0033 and $0.014 \mathrm{~dB} / \mathrm{km}$ at $S$ - and $C$-band, respectively [9]-[10]. Combining (13) and (14), we can obtain the cumulative differential phase corresponding to the $Z_{D R}$ bias due to differential attenuation. Using this procedure, we can obtain that when cumulative differential phase at $S$-band reaches $112^{\circ}$, then the bias in $Z_{D R}$ due to differential attenuation appears equivalent to $1-\mathrm{dB}$ error in $Z_{H}$. Similar $\Phi_{D P}$ value at $C$-band is $35^{\circ}$. Thus, we see that $Z_{D R}$ values have to be corrected for differential attenuation at $C$-band, whereas at $S$-band, it is needed when we use data over a long path, resulting in cumulative $\Phi_{D P}$ of over $112^{\circ}$.

\section{B. Error Structure of $K_{D P}$ and Calibration Bias Estimates}

The knowledge of the error structure of $K_{D P}, K_{D P}^{*}$ and bias is critical to the understanding of accuracy of the calibration procedure. Theoretical estimates of the standard errors in $K_{D P}, K_{D P}^{*}$ and bias in calibration are obtained in this section for a uniform precipitation path.

When the reflectivity factor is uniform along the rainfall path, we can estimate the mean value of the specific differential phase shift $K_{D P}$ through the least-squares fit procedure as

$$
\hat{K}_{D P}=\frac{\sum_{i=1}^{N}\left[\left(\Phi_{D P}\right)_{i}-\bar{\Phi}_{D P}\right]\left(r_{i}-\bar{r}\right)}{2 \sum_{i=1}^{N}\left(r_{i}-\bar{r}\right)^{2}}
$$

where $\hat{K}_{D P}$ is the estimate of $K_{D P},\left(\Phi_{D P}\right)_{i}$ is the two-way cumulative differential phase shift at the range bin $i, \bar{\Phi}_{D P}$ is the mean value of $\Phi_{D P}$ along the path, $r_{i}$ is the distance of the range bin from the radar, $\bar{r}$ is the average value of the path length, and $N$ is the total number of range bins. Assuming $r_{i}=i \Delta r$, where $\Delta r$ is the range resolution it can be easily shown that

$$
\begin{aligned}
\bar{r} & =\left(\frac{N+1}{2}\right) \Delta r \\
\sum_{i=1}^{N}\left(r_{i}-\bar{r}\right)^{2} & =\left[\frac{N(N-1)(N+1)}{12}\right] \Delta r^{2} .
\end{aligned}
$$

Substituting (16) in (15), we get

$$
\hat{K}_{D P}=\frac{\sum_{i=1}^{N}\left[\left(\Phi_{D P}\right)_{i}-\bar{\Phi}_{D P}\right]\left(i-\frac{N+1}{2}\right) \Delta r}{2 \frac{N(N-1)(N+1)}{12} \Delta r} .
$$

Subsequently, the variance of $\hat{K}_{D P}$ can be obtained as

$$
\begin{aligned}
\operatorname{var}\left(\hat{K}_{D P}\right) & =\operatorname{var}\left(\Phi_{D P}\right) \frac{\sum_{i=1}^{N}\left[\left(i-\frac{N+1}{2}\right) \Delta r\right]^{2}}{4\left[\frac{N(N-1)(N+1)}{12} \Delta r^{2}\right]^{2}} \\
& =\frac{\operatorname{var}\left(\Phi_{D P}\right)}{4 \frac{N(N-1)(N+1)}{12} \Delta r^{2}} .
\end{aligned}
$$

We can note here that (18) converges to (5), given by Ryzhkov and Zrnic [11], for large $N$. It can be observed from (18) that the variance of $\hat{K}_{D P}$ decreases by increasing the number of range bins, utilized in the estimate of $K_{D P}$, and the radial resolution $\Delta r$.

Following a similar procedure as above, we can estimate the mean value of $K_{D P}^{*}$ through the least-squares fit as

$$
\hat{K}_{D P}^{*}=\frac{\sum_{i=1}^{N}\left[\left(\Phi_{D P}^{*}\right)_{i}-\bar{\Phi}^{*}{ }_{D P}\right]\left(i-\frac{N+1}{2}\right) \Delta r}{2 \sum_{i=1}^{N}\left[\left(i-\frac{N+1}{2}\right) \Delta r\right]^{2}}
$$

where the $\left(\Phi_{D P}^{*}\right)_{i}$ can be written

$$
\left(\Phi_{D P}^{*}\right)_{i}=2\left(K_{D P}^{*}\right)_{i} i \Delta r .
$$


With simple algebraic manipulations, (19) can be reduced to

$$
\hat{K}_{D P}^{*}=\frac{\sum_{i=1}^{N}\left(K_{D P}^{*}\right)_{i}\left[\left(i-\frac{N+1}{2}\right) \Delta r\right]^{2}}{\sum_{i=1}^{N}\left[\left(i-\frac{N+1}{2}\right) \Delta r\right]^{2}} .
$$

The variance of the estimate (6) becomes [12]

$$
\operatorname{var}\left(\hat{K}_{D P}^{*}\right)=\frac{\sum_{i=1}^{N} \operatorname{var}\left(K_{D P}^{*}\right)_{i}\left[\left(i-\frac{N+1}{2}\right) \Delta r\right]^{4}}{\left[\sum_{i=1}^{N}\left(i-\frac{N+1}{2}\right)^{2} \Delta r^{2}\right]^{2}} .
$$

In each range bin, $\operatorname{var}\left(K_{D P}^{*}\right)$ is the same and can be written [3]

$$
\operatorname{var}\left(K_{D P}^{*}\right)=\left(K_{D P}^{*}\right)^{2} \nu
$$

where

$$
\nu=\left[0.053 \alpha^{2} \operatorname{var}\left(10 \log Z_{H}\right)+5.3 \beta^{2} \operatorname{var}\left(Z_{D R}\right)\right] .
$$

Then (22) can be expressed as

$$
\operatorname{var}\left(\hat{K}_{D P}^{*}\right)=\left(K_{D P}^{*}\right)^{2} \nu \frac{\sum_{i=1}^{N}\left[\left(i-\frac{N+1}{2}\right) \Delta r\right]^{4}}{\left[\sum_{i=1}^{N}\left(i-\frac{N+1}{2}\right)^{2}\right]^{2} \Delta r^{4}} .
$$

We want to point out that, unlike the variance of $\hat{K}_{D P}$, the variance of $\hat{K}_{D P}^{*}$ is independent of the range resolution $\Delta r$; moreover, it decreases slowly by increasing the number of the averaged range bins.

The parameters assumed for the computation of the radar measurement error are as follows:

1) radar frequency $3 \mathrm{GHz}$;

2) pulse repetition time $1 \mathrm{~ms}$;

3) number of sample pairs 64 ;

4) Doppler spectrum width $2 \mathrm{~m} / \mathrm{s}$;

5) zero lag cross-correlation coefficient 0.99 ;

6) range resolution $0.3 \mathrm{~km}$.

With those assumptions, we obtain the standard deviations in the estimates of $Z_{H}, Z_{D R}$, and $\Phi_{D P}$ to be $0.7 \mathrm{~dB}, 0.15 \mathrm{~dB}$, and $1^{\circ}$, respectively.

Fig. 4 shows the standard deviation of the estimates $\hat{K}_{D P}^{*}$ and $\hat{K}_{D P}$ for different values of $Z_{H}$. The $Z_{D R}$ value that corresponds to each $Z_{H}$ shown in Fig. 4 was chosen to be the average value of a $Z_{H}, Z_{D R}$ scatterplot in rainfall [13]. The $Z_{D R}$ values corresponding to the reflectivity values $30,35,40$. 45,50 , and $53 \mathrm{dBZ}$ shown in Fig. 4 are 0.9, 0.95, 1.3, 1.6, 1.9, and $2.0 \mathrm{~dB}$, respectively. We need to note here that the standard deviation of $\hat{K}_{D P}$ does not change with reflectivity. Figs. 5 and 6 show the fractional standard error (FSE) in the estimates $\hat{K}_{D P}$ and $\hat{K}_{D P}^{*}$ as a function of number of range bins over which the least-squares estimates are computed. It should be noted here that the various curves in Fig. 5 correspond to the same $Z_{H}, Z_{D R}$ pair shown in Fig. 4.

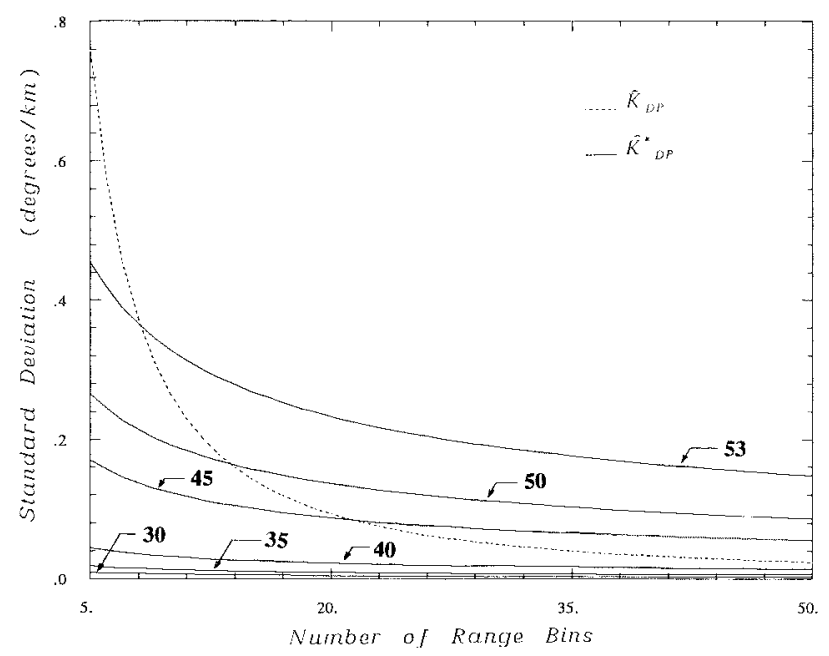

Fig. 4. Standard deviation of the estimate $\hat{K}_{D P}^{*}$, for different values of $Z_{H}$ (continuous lines), and of the estimate $\hat{K}_{D P}$ (dashed line) as a function of number of range bins.



Fig. 5. Fractional standard error of the estimate $\hat{K}_{D P}$ for different values of $Z_{H}$ as a function of number of range bins.



Fig. 6. Fractional standard error of the estimate $\hat{K}_{D P}^{*}$ as a function of number of range bins. 
The slope $s$ of the scatter diagram between $\hat{K}_{D P}^{*}$ and $\hat{K}_{D P}$ is utilized to estimate the bias in calibration. The least-squares estimate of the slope of a scattergram between the estimates $\hat{K}_{D P}^{*}$ and $\hat{K}_{D P}$ is given by

$$
\hat{s}=\frac{\sum_{i=1}^{M}\left(\hat{K}_{D P}^{*}\right)_{i}\left(\hat{K}_{D P}\right)_{i}}{\sum_{i=1}^{M}\left[\left(\hat{K}_{D P}^{*}\right)_{i}\right]^{2}}
$$

where $M$ is the number of the estimates. The variance in the estimate of the slope can be written as

$$
\operatorname{var}(\hat{s})=\frac{1}{M} \frac{\operatorname{var}\left(\hat{K}_{D P}^{*}\right)}{\operatorname{var}\left(\hat{K}_{D P}\right)}
$$

where $\rho$ is the correlation between the estimates of $K_{D P}^{*}$ and $K_{D P}$ [14]. The value of $\rho$ is very close to unity, typically of the order of 0.99 , as shown through simulation in the next section. It can be seen from Fig. 4 that the ratio of the standard deviation of the two $K_{D P}$ estimates commonly takes values between 0.1 and two. Therefore, the standard deviation of $\hat{s}$ can be approximated to be $0.3 / \sqrt{M}$. Thus, if we take about 100 estimates of $K_{D P}^{*}$ and $K_{D P}$ in moderate to heavy rain, the bias can be estimated to an accuracy of about $0.1 \mathrm{~dB}$. We need to note here that this estimate of accuracy in bias will vary depending on the accuracy of the two $K_{D P}$ estimates, which in turn depend on the path length over which $K_{D P}^{*}$ and $K_{D P}$ are estimated. Nevertheless, this first approximation provides an idea about the accuracy of the slope estimate.

\section{Simulation Study of Rain Profiles}

The various range profiles of $\Phi_{D P}, Z_{H}$, and $Z_{D R}$ are constructed by simulation with the following characteristics:

1) total path length of $90 \mathrm{~km}$;

2) range resolution $300 \mathrm{~m}$;

3) gradients of reflectivity in range vary between 0.5 and $50 \mathrm{~dB} / \mathrm{km}$;

4) maximum reflectivity along the path is less than $55 \mathrm{dBZ}$.

The reflectivity profile of the precipitation path is constructed such that, when the reflectivity is equal to $55 \mathrm{dBZ}$ or drops below $0 \mathrm{dBZ}$, the direction of the gradient is changed. Once the reflectivity is fixed at a range resolution volume, the parameters of the RSD, namely, $N_{0}, D_{0}$, and $\mu$, are chosen randomly under the constraint that the RSD yields the current reflectivity value in the range resolution cell. Subsequently, the values of $Z_{D R}$ and $K_{D P}$ are computed for the range location. This procedure yields a wide variety of range profiles of $Z_{H}, Z_{D R}$, and $K_{D P}$ so that the technique to evaluate calibration bias can be tested under all conditions. The value of $\Phi_{D P}(r)$ over range is computed from $K_{D P}$ as $2 \sigma K_{D P}(r) \Delta r$, where $\Delta r$ is the range resolution. Once the mean values of $Z_{H}, Z_{D R}$, and $\Phi_{D P}$ are fixed at each range resolution cell, the radar estimates with measurement error are simulated using the procedure given by Chandrasekar et al. [15].

Fig. 7 shows sample profiles of $Z_{H}, \Phi_{D P}$, and $\Phi_{D P}^{*}$ that are used in the simulation. There are several ways to compare the true and parameterized values of $K_{D P}$. However, practical constraints, such as error structure and range cumulative nature

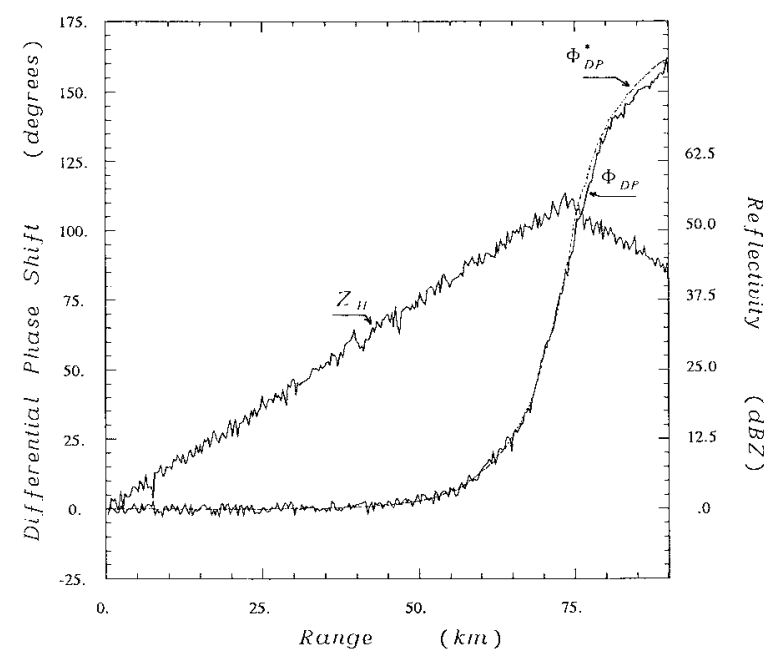

Fig. 7. Sample profiles of $Z_{H}, \Phi_{D P}$, and $\Phi_{D P}^{*}$ obtained in simulation as a function of range.

of differential propagation phase measurements, restrict the possibilities. In our analysis, $K_{D P}$ is estimated as the slope of the range profile of $\Phi_{D P}$ to minimize its standard error. This procedure has advantages and disadvantages. Using a least-squares fit to the $\Phi_{D P}$ profile, we can estimate $K_{D P}$ accurately in the case of a fairly long path (at least 15-20 range gates). However, when the path over which the least-squares fit is done becomes long, $K_{D P}$ cannot be uniform anymore within the path and the estimates are affected by gradients. When there are gradients in the path over which $K_{D P}$ is estimated, a bias is introduced in the $K_{D P}$ estimate [16]. In the context of estimating bias in the calibration of the radar system, even small errors in the estimate of $K_{D P}$ can be significant. To circumvent this problem, we apply an identical least-squares estimate to the parameterized $\Phi_{D P}^{*}$ profile. Therefore, an average $K_{D P}^{*}$ estimate over the path is obtained performing a least-squares fit to the $\Phi_{D P}^{*}$ profile. This procedure is adopted so that if there is a bias in the estimate of $K_{D P}$ due to gradients in the path, the same bias will be there in the estimate of $K_{D P}^{*}$. Thus, any difference between $K_{D P}$ and $K_{D P}^{*}$ will be due only to the bias in absolute calibration of the radar. Fig. 8 shows the scatterplot between the estimates of $K_{D P}$ and $K_{D P}^{*}$ obtained as slopes of $\Phi_{D P}$ and $\Phi_{D P}^{*}$ profiles, respectively. The various points correspond to the different paths considered in our simulation study. We can see that the scatter between the two estimates lies about at $45^{\circ}$. The slope of the scatter is 0.996 , and the standard deviation in the estimate of the slope is 0.007. This slope corresponds to a bias of $0.02 \mathrm{~dB}$. We can see that the accuracy of slope and bias estimates obtained from simulation are in the approximate range given theoretically from (27). Fig. 9 shows a similar scatterplot, except that the simulation includes a calibration bias of $1 \mathrm{~dB}$. The estimated slope of the scatter in Fig. 9 is 0.7926 , corresponding to a bias of $-1.05 \mathrm{~dB}$. The accuracy of the bias estimate depends on several factors, such as accuracy in measurements of $\Phi_{D P}, Z_{H}$, and $Z_{D R}$, path length in estimation of $K_{D P}$, and the number of data points in the scattergram of $\hat{K}_{D P}^{*}$ and $\hat{K}_{D P}$ estimates. Based on our simulations, it appears that, if we have sufficient data points 


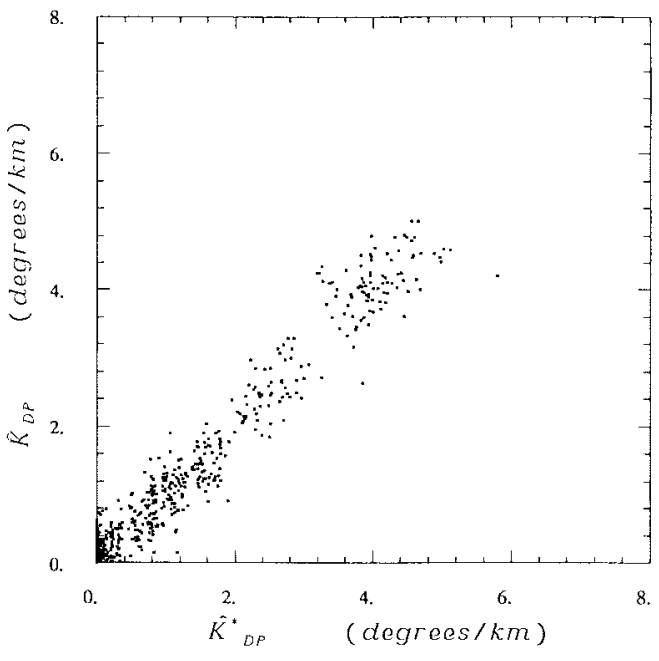

Fig. 8. Scatterplot between the estimates $\hat{K}_{D P}$ and $\hat{K}_{D P}^{*}$ obtained as slopes of $\Phi_{D P}$ and $\Phi_{D P}^{*}$, respectively.

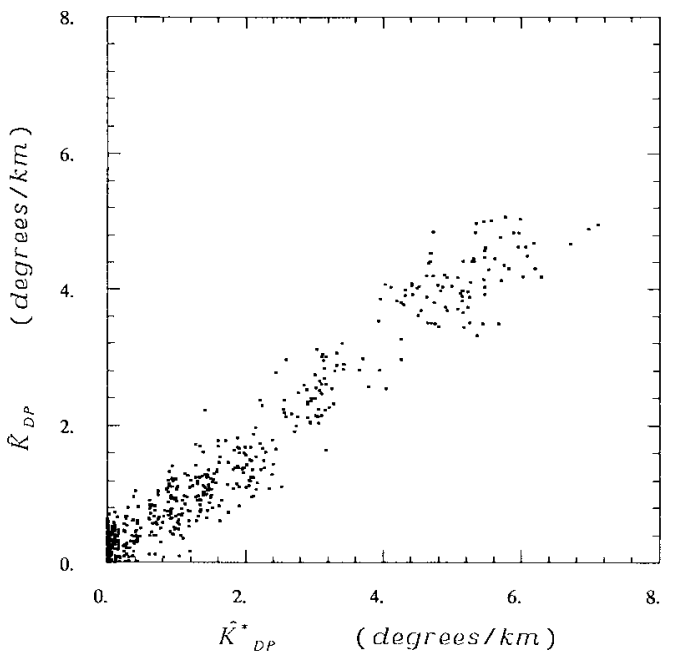

Fig. 9. Same as Fig. 9, introducing a bias of $1 \mathrm{~dB}$ in simulated $Z_{H}$ measurements.

for at least 100 estimates of $K_{D P}$, we can estimate the bias in the absolute calibration to an accuracy of a few tenth of $1 \mathrm{~dB}$. One important point to be noted here is the success of this procedure depends on obtaining many accurate estimates of $K_{D P}$ over various paths. Therefore, data from light rain (where there is not significant $K_{D P}$ ) should not be used in the calibration data set. A large amount of data from moderate to heavy rain $\left(>50 \mathrm{~mm} \mathrm{~h}^{-1}\right)$ will be very useful.

\section{Data Analysis}

Data collected during the CaPE experiment is used to validate the calibration procedure developed in this paper. During the later part of the CaPE experiment, the NCAR CP-2 radar was equipped with an auxiliary signal processor developed at Colorado State University to obtain differential phase measurements in addition to $Z_{H}$ and $Z_{D R}$. Table I shows the main features of the $\mathrm{CP}-2$ radar that are relevant to this paper. Fig. 10 shows a typical range profile of radar data used in the analysis. Fig. 11 shows the scatterplot between $\hat{K}_{D P}$ and $\hat{K}_{D P}^{*}$ estimates for data collected on September 29, 1991,
TABLE I

CHARACTERISTICS OF THE CP-2 RADAR

\begin{tabular}{l|c}
\hline \multicolumn{1}{c|}{ Characteristic } & CP-2 (S-Band) \\
\hline Polarization Type & linear \\
Wavelength (cm) & 10.7 \\
Peak Power $(\mathrm{kW})$ & 1200 \\
Pulse Length $(\mu s)$ & 1.0 \\
PRF $\left(s^{-1}\right)$ & 960 \\
Antenna Type & Center fed paraboloid \\
Antenna Size (m) & 8.5 \\
Beamwidth (degrees) & 0.93 \\
Polarizations transmitted & Linear V or H \\
Polarizations received & Copolar to transmit \\
Maximum Sidelobe level (dB) & -21 \\
Polarization Control Method & Ferrite switch \\
Polarization Control Period & Pulse by pulse \\
\hline
\end{tabular}

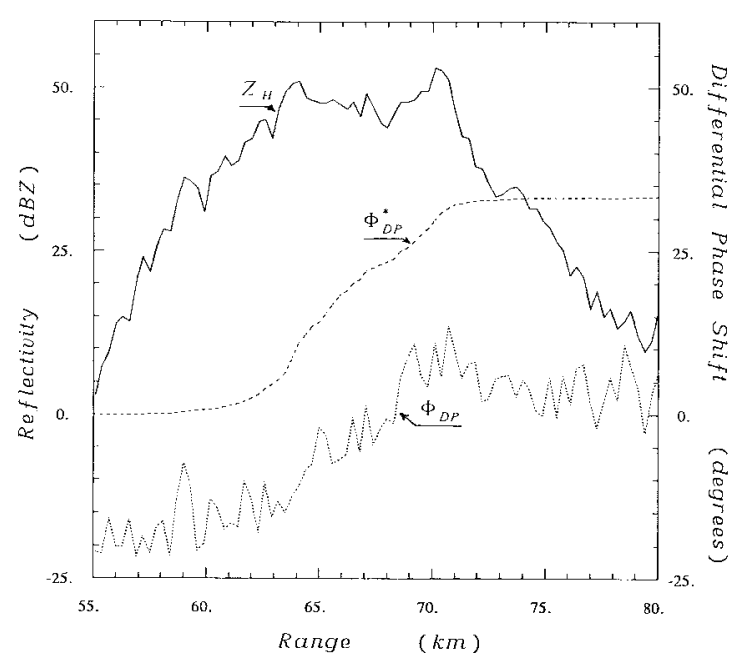

Fig. 10. Typical range profiles of $Z_{H}, \Phi_{D P}$, and $\Phi_{D P}^{*}$ obtained from radar data collected on September 29, 1991, by CP-2 during the CaPE experiment.

during the CaPE experiment. The measurements were obtained integrating 64 sample pairs, with a typical scan rate of 8 deg $\mathrm{s}^{-1}$. The $\hat{K}_{D P}$ estimates were obtained over 30 range gates. The scatterplot between $\hat{K}_{D P}$ and $\hat{K}_{D P}^{*}$ estimates has a deviation away from $45^{\circ}$ line that corresponds to a bias of $-1.1 \mathrm{~dB}$. Data collected over a long time during the day have shown the stability of the results. This bias determined from data was eventually traced to changes in system component characteristics in the radar.

\section{SUMMARY AND CONCLUSION}

The properties of shape and size distribution of raindrops translate into features of polarization diversity measurement. Polarization diversity observations in rain, namely, reflectivity, differential reflectivity, and specific differential propagation phase, lie in a constrained 3-D space. This self-consistency feature has been used to evaluate biases in the absolute calibration of the radar. In addition, vertical-looking observations in rainfall with antenna rotating about the vertical axis have been used to calibrate $Z_{D R}$ measurements. The selfconsistency feature of polarization diversity measurements are 


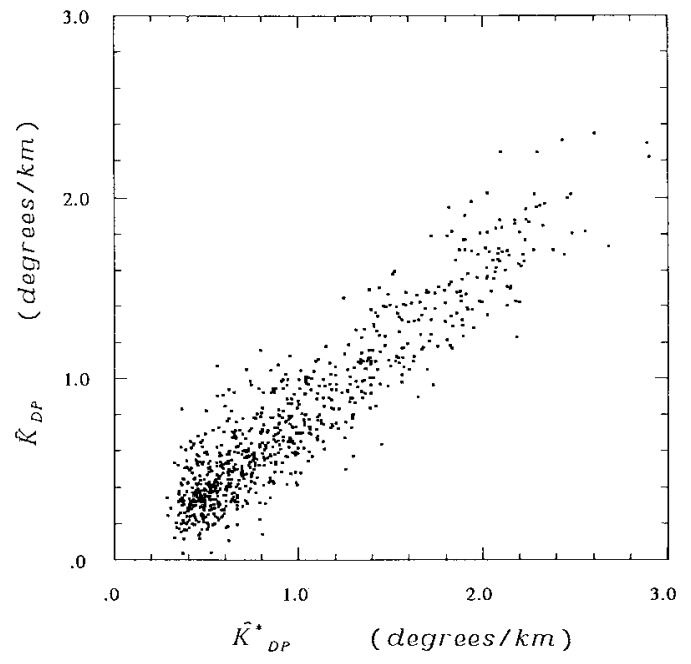

Fig. 11. Scatterplot between the estimates $\hat{K}_{D P}$ and $\hat{K}_{D P}^{*}$ obtained as slopes of $\Phi_{D P}$ and $\Phi_{D P}^{*}$, respectively. Radar data were collected on September 29 1991, by CP-2 during the CaPE experiment.

used to parameterize $K_{D P}$ as a function of reflectivity and differential reflectivity. Subsequently, this parameterized $K_{D P}^{*}$ is compared against directly measured $K_{D P}$ to evaluate the bias in the absolute calibration of the radar. Multiparameter radar measurements over a large number of precipitation paths were simulated to evaluate the feasibility of the procedure developed in this paper to estimate the bias in absolute calibration. Theoretical analysis as well as simulation study were used to demonstrate the sensitivity of the slope of scattergram between the estimates of $K_{D P}^{*}$ (involving selfconsistency principle) and measured $K_{D P}$ to biases in absolute calibration. A simple expression is provided to estimate the absolute calibration bias from the slope of the scattergram between $\hat{K}_{D P}^{*}$ and $\hat{K}_{D P}$ estimates.

The calibration procedure developed in this paper was applied to multiparameter radar data from $\mathrm{CaPE}$ experiment to evaluate the radar system bias. Data analysis and simulation study presented in this paper show that absolute calibration biases can be estimated to an accuracy less than $1 \mathrm{~dB}$. The actual accuracy depends on several factors, such as accuracies of $Z_{H}, Z_{D R}$, and $\Phi_{D P}$ measurements, path lengths over which $K_{D P}$ is computed from $\Phi_{D P}$, as well as the number of data points used in the estimates of $K_{D P}^{*}$ and $K_{D P}$. One important point to note here is that, to obtain good estimates of bias in absolute calibration, significant $\Phi_{D P}$ accumulation over the range profile has to be utilized. Data from light rain will not be sufficient to achieve this goal. Extensive data sets from moderate to heavy rainfall $\left(>50 \mathrm{~mm} \mathrm{~h}^{-1}\right)$ are needed to apply this procedure successfully.

\section{ACKNOWLEDGMENT}

The authors are grateful to Mr. P. Iacovelli for drafting and typing.

\section{REFERENCES}

[1] T. A Seliga, and V. N. Bringi, "Potential use of the reflectivity at orthogonal polarizations for measuring precipitation," J. Appl. Meteorol., vol. 15, pp. 69-76, 1976.
[2] V. Chandrasekar, W. A. Cooper, and V. N. Bringi, "Axis ratios and oscillation of raindrops," J. Atmos. Sci., vol. 45, pp. 1325-1333, 1988.

[3] G. Scarchilli, E. Gorgucci, V. Chandrasekar, and A. Dobaie, "Selfconsistency of polarization diversity measurement of rainfall," IEEE Trans. Geosci. Remote Sensing, vol. 34, pp. 22-26, Jan. 1996.

[4] V. Chandrasekar, V. N. Bringi, N. Balakrishnan, and D. S. Zrnic, "Error structure of multiparameter radar and surface measurements of precipitation, Part III: Propagation differential phase shift," J. Atmos. Ocean. Technol., vol. 7, pp. 621-629, 1990.

[5] C. W. Ulbrich, "Natural variations in the analytical form of raindrop size distributions," J. Clim. Appl. Meteorol., vol. 22, pp. 1764-1775, 1983.

[6] H. R. Pruppacher and R. L. Pitter, "A semi-empirical determination of the shape of cloud and raindrops," J. Atmos. Sci., vol. 28, pp. 86-94, 1971.

[7] G. B. Foote, "Scientific overview and operations plan," Nat. Center Atmos. Res., Boulder, CO, 1991, p. 145.

[8] A. B. Kostinski, "Fluctuations of differential phase and radar measurements of precipitation," J. Appl. Meteorol., vol. 33, pp. 1176-1181, 1994.

[9] V. N. Bringi, V. Chandrasekar, N. Balakrishnan, and D. S. Zrnic, "An examination of propagation effects in rainfall on radar measurements at microwave frequencies," J. Atmos. Ocean. Technol., vol. 7, pp. 829-840, 1990.

[10] G. Scarchilli, E. Gorgucci, V. Chandrasekar, and T. A. Seliga, "Rainfall estimation using polarimetric techniques at C-band frequencies," J. Appl. Meteorol., vol. 32, pp. 1150-1160, 1993.

[11] A. Ryzhkov and D. S. Zrnic, "Assessment of rainfall measurement that uses specific differential phase," J. Appl. Meteorol., vol. 35, pp. 2080-2090, 1996.

[12] A. Papoulis, Probability, Random Variables, and Stochastic Processes. New York: McGraw-Hill, 1965, p. 583.

[13] V. N. Bringi, V. Chandrasekar, P. Meischner, J. Hubbert, and Y. Golestani, "Polarimetric radar signatures of precipitation at S- and C-bands," Proc. Inst. Elect. Eng. F, vol. 138, no. 2, pp. 109-115, 1991.

[14] M. G. Kendall and A. Stuart, The Advanced Theory of Statistics, vol. 2. New York: Griffin, 1973, p. 723.

[15] V. Chandrasekar, V. N. Bringi, and P. J. Brockwell, "Statistical properties of dual polarized radar signals," preprints Proc. 23th Conf. Radar Meteorol. Snowmass, CO: Amer. Meteorol. Soc., 1986, pp. 154-157.

[16] G. Scarchilli, E. Gorgucci, and V. Chandrasekar, "Influence of reflectivity on $\Phi_{D P}$ and $K_{D P}$ estimate," preprints Proc. 26th Conf. Radar Meteorol. Norman, OK: Amer. Meteorol. Soc., 1993, pp. 103-105.

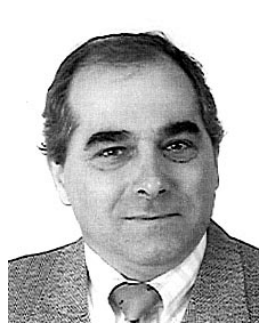

Eugenio Gorgucci received the doctoral degree in physics from La Sapienza University, Rome, Italy.

He has been with the Institute of Atmospheric Physics (IFA), National Research Council (CNR), Rome, as a Research Scientist since 1976. His research interests include radar meteorology, signal processing, wave propagation, and scattering.

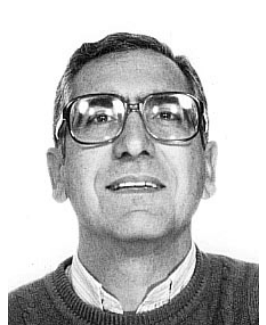

Gianfranco Scarchilli received the doctoral degree in electronics engineering from La Sapienza University, Rome, Italy.

$\mathrm{He}$ is with the Institute of Atmospheric Physics (IFA) of the National Research Council (CNR), Rome, where he is a Research Scientist. His research interests include radar meteorology, signal processing, and wave propagation.

V. Chandrasekar (S'83-M'83), photograph and biography not available at the time of publication. 